\title{
Olivier's theorem: ideal convergence, algebrability and Borel classification
}

\section{Artur Bartoszewicz ${ }^{1}$ (D) Szymon Głąb $^{2}$ (D) Agnieszka Widz ${ }^{2}$}

Received: 1 March 2021 / Accepted: 7 September 2021

(c) The Author(s) 2021, corrected publication 2021

\begin{abstract}
The classical Olivier's theorem says that for any nonincreasing summable sequence $(a(n))$ the sequence $(n a(n))$ tends to zero. This result was generalized by many authors. We propose its further generalization which implies known results. Next we consider the subset $\mathcal{A O S}$ of $\ell_{1}$ consisting of sequences for which the assertion of Olivier's theorem is false. We study how large and good algebraic structures are contained in $\mathcal{A O S}$ and its subsets; this kind of study is known as lineability. Finally we show that $\mathcal{A O S}$ is a residual $\mathcal{G}_{\delta \sigma}$ but not an $\mathcal{F}_{\sigma \delta}$-set.
\end{abstract}

Keywords Olivier's theorem · Algebrability $\cdot$ Spaceability $\cdot$ Ideal convergence $\cdot$ True $G_{\delta \sigma}$ sets

Mathematics Subject Classification $15 \mathrm{~A} 03 \cdot 40 \mathrm{~A} 05 \cdot 54 \mathrm{H} 05 \cdot 40 \mathrm{~A} 35$

\section{Introduction}

Throughout the paper by $\mathbf{a}=(a(n)), \mathbf{b}=(b(n))$, etc. we denote sequences of reals. The sequences of positive integer numbers are denoted by $\left(n_{k}\right),\left(m_{k}\right)$ and so one.

It is a very basic convergence test, that for convergent series $\sum a(n)$ the limit of its summands $\lim _{n \rightarrow \infty} a(n)$ is zero. However, the following classical Olivier's theorem seems a bit surprising for many mathematicians.

Theorem 1.1 [13] For any nonincreasing summable sequence $\mathbf{a}=(a(n))$ the sequence $(n a(n))$ tends to zero.

Szymon Głąb

szymon.glab@p.lodz.pl

Artur Bartoszewicz

artur.bartoszewicz@wmii.uni.lodz.pl

Agnieszka Widz

agnieszkawidzenfp@gmail.com

1 Faculty of Mathematics and Computer Science, Łódź University, ul. Stefana Banacha 22, 90-238 Łódź, Poland

2 Institute of Mathematics, Łódź University of Technology, ul. Wólczańska 215, 93-005 Lodz, Poland 
Krzyż [10] has written that the following generalization of the Olivier's theorem can be found in [15].

Theorem 1.2 For any nonincreasing sequence $\mathbf{a}=(a(n))$ tending to zero and for a sequence $\boldsymbol{\mu}=(\mu(n))$ for which $\sum a(n) \mu(n)<\infty$, we have

$$
\lim _{n \rightarrow \infty}(\mu(1)+\cdots+\mu(n)) a(n)=0 .
$$

Putting $\mu(n)=1$ we obtain the classic Olivier's theorem. It is not difficult to see that the assumption of the monotonicity of the sequence $\mathbf{a}$ is essential in both theorems.

Example 1.1 Let $a(n)=\frac{1}{n}$ for $n=k^{2}$ where $k=1,2, \ldots$ and $a(n)=\frac{1}{2^{n}}$ for other numbers $n$. Then $\sum a(n)<\infty$ but $\lim _{k \rightarrow \infty} k^{2} a\left(k^{2}\right)=1$. It was observed in [14] that a subsequence $(n a(n))$ for which $n a(n)$ does not tend to zero can be unbounded.

Many authors have tried to omit the assumption of monotonicity in different ways. For example the authors of [7] have found the full characterization of sequences $(a(n))$ for which the assertion of the Olivier's theorem holds. The second way is to consider the ideal convergence instead of the usual convergence of sequences. Recall that for an admissible ideal $\mathcal{I}$ on $\mathbb{N}$, where admissible means containing finite sets and non-equal to $\mathcal{P}(\mathbb{N})$, we have $\mathcal{I}$ - $\lim a(n)=0$ or $a(n) \stackrel{\mathcal{I}}{\rightarrow} 0$ if and only if for any $\varepsilon>0$ the set $A_{\varepsilon}=\{n:|a(n)| \geq \epsilon\}$ belongs to $\mathcal{I}$, see for example [9]. Clearly for $\mathcal{I}=$ Fin (the ideal of finite sets) the ideal convergence reduces to the usual convergence. The main result in [14] is the following.

Theorem 1.3 Let $\mathbf{a}=(a(n))$ be an arbitrary sequence of nonnegative numbers. Then the convergence of the series $\sum a(n)$ implies the $\mathcal{I}$-convergence to zero of the sequence $(n a(n))$ if and only if an ideal $\mathcal{I}$ contains the summable ideal $\mathcal{I}_{\left(\frac{1}{n}\right)}$.

Recall that an ideal $\mathcal{I}_{(x)}$ consists of the sets $A \subset \mathbb{N}$ for which $\sum_{n \in A} x(n)<\infty$ for divergent series $\sum x(n)=\infty$ with $x(n) \geq 0$. Note that for a summable sequence $\mathbf{x}=x(n)$ the ideal $\mathcal{I}_{(x)}=\mathcal{P}(\mathbb{N})$, so it is not admissible. If there exists $c>0$ such that we have $x(n)>c$ for all $n \in \mathbb{N}$, then $\mathcal{I}_{(x)}=$ Fin. The result of Toma and Šalát was further generalized in [8] where the authors characterized ideals $\mathcal{I}$ for which the implication

$$
\sum a^{p}(n)<\infty \Rightarrow n a(n) \stackrel{\mathcal{I}}{\rightarrow} 0
$$

holds true for a fixed positive $p$.

Recently Mišík and Tóth in [12] have proved the following theorem that generalizes those of $[8,14]$.

Theorem 1.4 Let $p, q$ be fixed positive numbers and $\alpha, \beta$ be fixed nonnegative ones. Moreover, let $\mathbf{a}=(a(n))$ be an arbitrary sequence with positive terms. Then the convergence of the series $\sum n^{\alpha} a^{p}(n)$ implies the $\mathcal{I}$-convergence to zero of the sequence $\left(n^{\beta} a^{q}(n)\right)$ if and only if an ideal $\mathcal{I}$ contains the summable ideal $\mathcal{I}_{\left(n^{\alpha-\beta p / q)}\right.}$.

The paper is organized as follows. The main result of Sect. 2 further generalizes Theorem 1.4. We show that it implies several known results and it cannot be inferred from them. Next we consider the subset of $\ell_{1}$ consisting of sequences for which the assertion of Olivier's theorem is false. We shall call such sequences Anti-Olivier and the set of all them denote by $\mathcal{A O S}$. In fact we consider the algebraic and topological properties of some subsets of $\mathcal{A O S}$. In Sect. 3 we study how large and good algebraic structures are contained in $\mathcal{A O S}$ and its subsets; this kind of study is known as lineability, see [1,5]. Section 4 is devoted to the Borel classification of considered sets in $\ell_{1}$. The main result here is that $\mathcal{A O S}$ is a residual $\mathcal{G}_{\delta \sigma}$ but not an $\mathcal{F}_{\sigma \delta}$-set. 


\section{Olivier's theorem and the ideal convergence}

Theorem 2.1 Let $\boldsymbol{f}, \boldsymbol{g}: \mathbb{N} \rightarrow \mathbb{R}^{+}$and let $p, q$ be fixed positive numbers. The following conditions are equivalent:

(1) For any sequence $\mathbf{a}=(a(n))$ of positive numbers the convergence of series $\sum f(n) a^{p}(n)$ implies that $g(n) a^{q}(n) \stackrel{\mathcal{I}}{\rightarrow} 0$.

(2) $\mathcal{I}_{\left(f g^{-p / q}\right)} \subset \mathcal{I}$, where $\mathcal{I}_{\left(f g^{-p / q}\right)}=\left\{A \subset \mathbb{N}: \sum_{n \in A} f(n) g^{-p / q}(n)<\infty\right\}$ is a summable ideal.

Proof $(2) \Rightarrow(1)$

Let us assume that $\mathcal{I}_{\left(f g^{-p / q}\right)} \subset \mathcal{I}$ and $\sum f(n) a^{p}(n)<\infty$. We shall show that $g(n) a^{q}(n) \stackrel{\mathcal{I}}{\rightarrow} 0$. Namely, we need to prove that

$$
\forall \varepsilon>0\left\{n \in \mathbb{N}: g(n) a^{q}(n) \geq \varepsilon\right\} \in \mathcal{I} .
$$

Let us fix $\varepsilon>0$. Since $p, q, f$ and $g$ are positive, we have

$$
\begin{aligned}
A_{\varepsilon} & :=\left\{n \in \mathbb{N}: g(n) a^{q}(n) \geq \varepsilon\right\} \\
& =\left\{n \in \mathbb{N}: g^{\frac{p}{q}}(n) a^{p}(n) \geq \varepsilon^{\frac{p}{q}}\right\} \\
& =\left\{n \in \mathbb{N}: \varepsilon^{-\frac{p}{q}} f(n) a^{p}(n) \geq \frac{f(n)}{g^{\frac{p}{q}}(n)}\right\} .
\end{aligned}
$$

We have

$$
\sum_{n \in A_{\varepsilon}} \frac{f(n)}{g^{\frac{p}{q}}(n)} \leq \sum_{n \in A_{\varepsilon}} \varepsilon^{-\frac{p}{q}} f(n) a^{p}(n)=\varepsilon^{-\frac{p}{q}} \sum_{n \in A_{\varepsilon}} f(n) a^{p}(n)<\infty .
$$

Hence $A_{\varepsilon} \in \mathcal{I}_{\left(f g^{-p / q}\right)}$. Since $\mathcal{I}_{\left(f g^{-p / q}\right)} \subset \mathcal{I}$, then $A_{\varepsilon} \in \mathcal{I}$ and $g(n) a^{q}(n) \stackrel{\mathcal{I}}{\rightarrow} 0$.

(1) $\Rightarrow(2)$

Let us assume that the implication

$$
\sum f(n) a^{p}(n)<\infty \Rightarrow g(n) a^{q}(n) \stackrel{\mathcal{I}}{\rightarrow} 0
$$

holds for any sequence a of positive numbers. Let us put $A_{\mathbf{a}, \varepsilon}:=\left\{n \in \mathbb{N}: g(n) a^{q}(n) \geq \varepsilon\right\}$. Now, our assumption can be written as follows

$$
\sum f(n) a^{p}(n)<\infty \Rightarrow \forall \varepsilon>0 \quad A_{\mathbf{a}, \varepsilon} \in \mathcal{I} .
$$

We will show that $\mathcal{I}_{\left(f g^{-p / q)}\right.} \subset \mathcal{I}$. To do this, we will show that any set $B$ belonging to the ideal $\mathcal{I}_{\left(f g^{-p / q)}\right.}$ is a subset of $A_{\mathbf{a}, \varepsilon}$ for some $\varepsilon>0$ and some sequence a of positive numbers, in symbols

$$
\forall B \in \mathcal{I}_{\left(f g^{-p / q}\right)} \exists \varepsilon>0 \exists \mathbf{a}\left(B \subset A_{\mathbf{a}, \varepsilon} \text { and } \sum f(n) a^{p}(n)<\infty\right) .
$$

Let us take a set $B$ for which $\sum_{n \in B} f(n) / g^{\frac{p}{q}}(n)<\infty$. Let us define a as follows.

$$
a(n)= \begin{cases}\left(g^{\frac{1}{q}}(n)\right)^{-1} & \text { for } n \in B \\ \left(f^{\frac{1}{p}}(n) \cdot 2^{\frac{n}{p}}\right)^{-1} & \text { for } n \notin B .\end{cases}
$$


Then $\sum f(n) a^{p}(n)<\infty$. Indeed,

$$
\begin{aligned}
\sum f(n) a^{p}(n) & =\sum_{n \in B} \frac{f(n)}{g^{\frac{p}{q}}(n)}+\sum_{n \notin B} \frac{f(n)}{\left(f^{\frac{1}{p}}(n) \cdot 2^{\frac{n}{p}}\right)^{p}} \\
& =\sum_{n \in B} \frac{f(n)}{g^{\frac{p}{q}}(n)}+\sum_{n \notin B} \frac{1}{2^{n}}<\infty .
\end{aligned}
$$

Moreover, $B \subset A_{\mathbf{a}, 1}\left(A_{\mathbf{a}, \varepsilon}\right.$ for $\left.\varepsilon=1\right)$. Indeed,

$$
\begin{aligned}
A_{\mathbf{a}, 1} & =\left\{n \in \mathbb{N}: g(n) a^{q}(n) \geq 1\right\} \\
& =\left\{n \in B: g(n)\left(g(n)^{-\frac{1}{q}}\right)^{q} \geq 1\right\} \cup\left\{n \notin B: g(n)\left(f^{-\frac{1}{p}}(n) 2^{-\frac{n}{p}}\right)^{q} \geq 1\right\} \supset B .
\end{aligned}
$$

Hence, an arbitrary set $B \in \mathcal{I}_{(f g-p / q)}$ is contained in $A_{\mathbf{a}, \varepsilon}$ for $\varepsilon=1$ and for some sequence a for which $\sum f(n) a^{p}(n)<\infty$. Hence $B \in \mathcal{I}$ and consequently $\mathcal{I}_{\left(f g^{-p / q}\right)} \subset \mathcal{I}$.

Let us consider some particular cases of the Theorem 2.1.

- Putting $f(n)=1, g(n)=1, p=q=1$. We obtain that $\sum a(n)<\infty \Rightarrow a(n) \stackrel{\mathcal{I}}{\longrightarrow} 0$ if and only if Fin $\subset \mathcal{I}$. This is equivalent to a basic convergence test: $\sum a(n)<\infty$ implies $\lim _{n \rightarrow \infty} a(n)=0$.

- Putting $f(n)=\mu(n), g(n)=\mu(1)+\cdots+\mu(n), p=q=1$, we obtain the ideal version of Theorem 1.2.

- Putting $f(n)=1, g(n)=n, p=q=1$, we obtain Theorem 1.3.

- Putting $f(n)=n^{\alpha}, g(n)=n^{\beta}$, we obtain Theorem 1.4.

- Putting $f(n)=b(n), g(n)=1$ and $p=q=1$, we obtain that $\sum a(n) b(n)<\infty$ implies $a(n) \stackrel{\mathcal{I}_{(b)}}{\longrightarrow} 0$ and similarly $b(n) \stackrel{\mathcal{I}_{(a)}}{\longrightarrow} 0$.

\section{Algebraic structures in AOS and its subsets}

Having an algebraic structure $A$ and its subset $E \subset A$ one can ask if $E \cup\{\mathbf{0}\}$ contains a large substructure of $A$. Roughly speaking, if the answer is affirmative, then we say that $E$ is structurable. Let $\kappa$ be a cardinal number. Assume that $V$ is a linear space (resp. an algebra). A subset $E \subset V$ is called $\kappa$-lineable (resp. $\kappa$-algebrable) whenever $E \cup\{\boldsymbol{0}\}$ contains an $\kappa$-dimensional subspace (resp. $\kappa$-generated algebra). We say that an algebra is $\kappa$-generated if the minimal cardinality of sets of its generators is equal to $\kappa$. Moreover, we say that a subset $E$ of an algebra $V$ is strongly $\kappa$-algebrable [4] if there exists algebra $A \subset E \cup\{\mathbf{0}\}$ which is isomorphic with the $\kappa$-generated free algebra.

Let us recall that $X=\left\{x_{\alpha}: \alpha<\kappa\right\} \subset E$ is a set of free generators of a free algebra $A \subset E$ if the set $X^{\prime}$ of elements of the form $x_{\alpha_{1}}^{k_{1}} \ldots x_{\alpha_{n}}^{k_{n}}$ is linearly independent and all linear combinations of elements from $X^{\prime}$ are in $E \cup\{\mathbf{0}\}$. It means that for any nonzero polynomial $P$ in $n$ variables without a constant term and any distinct $x_{\alpha_{1}}, \ldots, x_{\alpha_{n}} \in X$ we have $0 \neq P\left(x_{\alpha_{1}}, \ldots, x_{\alpha_{n}}\right) \in E$. Let $B$ be a Banach space. A subset $M \subset B$ is called spaceable if $M \cup\{\boldsymbol{0}\}$ contains an infinitely dimensional closed subspace $Y$ of $B$. We have the following obvious implications:

$$
\begin{aligned}
\kappa \text {-strong algebrability } & \Rightarrow \kappa \text {-lineability } \\
\text { spaceability } & \Rightarrow \mathfrak{c} \text {-lineability. }
\end{aligned}
$$


More information and more examples on these notions can be found in survey article [5] and book [1].

Let us define the following subsets of $\ell_{1}$ strictly connected with Olivier's Theorem.

- Set of AntiOlivier sequences $\mathcal{A O S}=\left\{\mathbf{a} \in \ell_{1}: n a(n) \nrightarrow 0\right\}$

- Set of sequences with finite number of terms equal to zero:

$$
\mathcal{F} \mathcal{Z}=\left\{\mathbf{a} \in \ell_{1}: \exists k \forall n>k \quad a(n) \neq 0\right\} .
$$

- $\mathcal{A O S}_{b}=\left\{\mathbf{a} \in \ell_{1}: n a(n) \nrightarrow 0\right.$ and $(n a(n))_{n=1}^{\infty}$ is bounded $\}$

- $\mathcal{A O S}_{u b}=\left\{\mathbf{a} \in \ell_{1}: \lim \sup |n(a(n))|=\infty\right\}$.

Let us observe that $\mathcal{A O S}_{b} \cap \mathcal{A O S}_{u b}=\emptyset$ and $\mathcal{A O S}_{b} \cup \mathcal{A O S} \mathcal{S}_{u b}=\mathcal{A O S}$.

Theorem 3.1 The set $\mathcal{A O S}_{b} \cap \mathcal{F Z}$ is c-lineable but it is not 1-algebrable.

Theorem 3.2 The set $\mathcal{A O S}_{u b} \cap \mathcal{F} \mathcal{Z}$ is strongly c-algebrable.

Theorem 3.3 The sets $A O S_{b}$ and $A O S_{u b}$ are spaceable.

Proof of Theorem 3.1 We say that a family $\mathcal{F}$ of subsets of $\mathbb{N}$ (or of another countable set) is almost disjoint if it consists of infinite sets and the intersection of any two distinct members of $\mathcal{F}$ is finite. Probably, Sierpiński was the first who observed that there exists an almost disjoint family $\mathcal{F}$ with the cardinality $2^{\omega}=\mathfrak{c}$.

Let $A \in \mathcal{I}_{(1 / n)}$ be infinite, $\Lambda \subseteq(2, \infty)$ be linearly independent over the field of rational numbers $\mathbb{Q}$ with $|\Lambda|=\mathfrak{c}$ and $\mathcal{F}=\left\{A_{\alpha}: \alpha \in \Lambda\right\}$ be an almost disjoint family of subsets of $A$ such that $\bigcup \mathcal{F}=A$. Let us define sequences $\mathbf{a}^{\alpha}, \alpha \in \Lambda$, by the formula:

$$
a^{\alpha}(n)= \begin{cases}\frac{1}{n} & \text { for } n \in A_{\alpha} \\ \frac{1}{n^{\alpha}} & \text { for } n \notin A_{\alpha} .\end{cases}
$$

Let us consider a linear combination $\mathbf{b}=\left(c_{1} \mathbf{a}^{\alpha_{1}}+\cdots+c_{k} \mathbf{a}^{\alpha_{k}}\right)$ for pairwise distinct $\alpha_{1}, \ldots, \alpha_{k}$ and $c_{i} \in \mathbb{R} \backslash\{0\}$, for $i \leq k$. Clearly $\mathbf{b} \in \ell_{1}$ as a linear combination of $\ell_{1}$-sequences.

Since $\mathcal{F}$ consists of almost disjoint sets, the set $\mathbb{N}$ is a disjoint union of $k+1$ infinite sets: $A_{\alpha_{i}} \backslash \bigcup_{j \leq k, j \neq i} A_{\alpha_{j}}$ for $i \leq k$, and $\mathbb{N} \backslash \bigcup_{i=1}^{k} A_{\alpha_{i}}$. We will show that the sequence $(n b(n))$ tends on $A_{\alpha_{i}} \backslash \bigcup_{j \neq i} A_{\alpha_{j}}$ to $c_{i} \neq 0$. This shows that the sequence $(n b(n))$ is bounded and at most finitely many $b(n)$ 's equal zero there. We will also show that $|b(n)|>0$ for almost every $n \in \mathbb{N} \backslash \bigcup_{i=1}^{k} A_{\alpha_{i}}$. Consequently, we will show that $\mathbf{b} \in \mathcal{A O S} \mathcal{S}_{b} \cap \mathcal{F} \mathcal{Z}$.

Let $n \in A_{\alpha_{1}} \backslash \bigcup_{j \geq 2} A_{\alpha_{j}}$ (if $n \in A_{\alpha_{i}} \backslash \bigcup_{j \neq i} A_{\alpha_{j}}$, the reasoning is the same). Then

$$
n(b(n))=n\left(c_{1} \frac{1}{n}+c_{2} \frac{1}{n^{\alpha_{2}}}+\cdots+c_{k} \frac{1}{n^{\alpha_{k}}}\right)=\left(c_{1}+c_{2} n^{1-\alpha_{2}}+\cdots+c_{k} n^{1-\alpha_{k}}\right) .
$$

Thus the sequence $(n(b(n)))$ tends to $c_{1} \neq 0$ along indices belonging to $A_{\alpha_{1}} \backslash \bigcup_{j \geq 2} A_{\alpha_{j}}$.

Let $n \notin \bigcup_{i=1}^{m} A_{\alpha_{i}}$. We may assume that $\alpha_{1}<\alpha_{2}<\cdots<\alpha_{k}$. Hence

$$
|b(n)|=\left|c_{1} \frac{1}{n^{\alpha_{1}}}+c_{2} \frac{1}{n^{\alpha_{2}}}+\cdots+c_{k} \frac{1}{n^{\alpha_{k}}}\right| \geq \frac{\left|c_{1}\right|}{n^{\alpha_{1}}}\left(1-\frac{\left|c_{2}\right|}{\left|c_{1}\right|} n^{\alpha_{1}-\alpha_{2}}-\cdots-\frac{\left|c_{k}\right|}{\left|c_{1}\right|} n^{\alpha_{1}-\alpha_{k}}\right)
$$

which is greater than zero for sufficiently large numbers $n$.

Finally note that the set $\mathcal{A O S}_{b}$ is not 1-algebrable. Indeed, assume that for all $n$ we have $|n a(n)|<M$. It means that $a(n)<M / n$ and hence $a^{2}(n)<M^{2} / n^{2}$. Therefore $n a^{2}(n) \rightarrow 0$, which means that $\mathbf{a}^{2} \notin \mathcal{A O S}$. 
Proof of Theorem 3.2 Let us define an increasing sequence of positive integers $m_{1}<m_{2}<$ ... such that

$$
m_{k}\left(\frac{1}{k}\right)^{k}>k
$$

It is enough to take $m_{k}:=k^{k+1}+1$. Let $\Lambda \subset(2, \infty)$ be a linearly independent set over rational with $|\Lambda|=\mathfrak{c}$. Denote the set $\left\{m_{1}, m_{2}, \ldots\right\}$ by $M$. Let us define sequences $\mathbf{a}^{\alpha}$ for $\alpha \in \Lambda$ by the formula

$$
a^{\alpha}(n)= \begin{cases}\frac{1}{k^{\alpha}} & \text { for } n=m_{k} \in M \\ \frac{1}{n^{\alpha}} & \text { for } n \notin M .\end{cases}
$$

Let $P\left(x_{1}, \ldots, x_{q}\right)$ be a polynomial in $q$ variables without a constant term. Hence $P$ is of the form $P\left(x_{1}, \ldots, x_{q}\right)=\sum_{i=1}^{p} c_{i} x_{1}^{\beta_{i 1}} \cdots x_{q}^{\beta_{i q}}$ where $B=\left[\beta_{i j}\right]_{\substack{1 \leq i \leq p \\ 1 \leq j \leq q}}$ is a matrix with nonnegative integer terms and with nonzero, pairwise distinct rows. Then, for $m_{k} \in M$ we have

$$
P\left(\mathbf{a}^{\alpha_{1}}, \ldots, \mathbf{a}^{\alpha_{q}}\right)\left(m_{k}\right)=\sum_{i=1}^{p} c_{i} k^{-\left(\alpha_{1} \beta_{i_{1}}+\cdots+\alpha_{q} \beta_{i_{q}}\right)}=\sum_{i=1}^{p} c_{i} k^{-r_{i}}
$$

where $r_{i}=\alpha_{1} \beta_{i_{1}}+\cdots+\alpha_{q} \beta_{i_{q}}$. Let us observe that by the linear independence of $\Lambda$ the numbers $r_{1}, r_{2}, \ldots, r_{p}$ are nonzero and pairwise distinct. We may assume that $r_{1}=$ $\min \left(r_{1}, r_{2}, \ldots, r_{p}\right)$. Hence,

$$
\begin{aligned}
m_{k}\left|P\left(\mathbf{a}^{\alpha_{1}}, \ldots, \mathbf{a}^{\alpha_{q}}\right)\left(m_{k}\right)\right| & =m_{k}\left|c_{1} k^{-r_{1}}\left(1+\frac{c_{2}}{c_{1}} k^{r_{1}-r_{2}}+\cdots+\frac{c_{p}}{c_{1}} k^{r_{1}-r_{p}}\right)\right| \\
& \geq m_{k}\left|c_{1}\right| k^{-r_{1}}\left(1-\left|\frac{c_{2}}{c_{1}}\right| k^{r_{1}-r_{2}}-\cdots-\left|\frac{c_{p}}{c_{1}}\right| k^{r_{1}-r_{p}}\right) .
\end{aligned}
$$

Since $r_{1}-r_{j}<0$, then for sufficiently large $k$

$$
m_{k}\left|P\left(\mathbf{a}^{\alpha_{1}}, \ldots, \mathbf{a}^{\alpha_{q}}\right)\left(m_{k}\right)\right| \geq \frac{m_{k}\left|c_{1}\right| k^{-r_{1}}}{2}>\frac{\left|c_{1}\right| k^{k+1-r_{1}}}{2} \rightarrow \infty .
$$

Hence $P\left(\mathbf{a}^{\alpha_{1}}, \ldots, \mathbf{a}^{\alpha_{q}}\right) \in \mathcal{A O S}_{u b}$ and $P\left(\mathbf{a}^{\alpha_{1}}, \ldots, \mathbf{a}^{\alpha_{q}}\right)(n) \neq 0$ for almost all $n \in M$. Then the same argument shows $P\left(\mathbf{a}^{\alpha_{1}}, \ldots, \mathbf{a}^{\alpha_{q}}\right)(n) \neq 0$ for all but finitely many numbers $n \in \mathbb{N} \backslash M$. Consequently $P\left(\mathbf{a}^{\alpha_{1}}, \ldots, \mathbf{a}^{\alpha_{q}}\right) \in \mathcal{F} \mathcal{Z}$.

Proof of Theorem 3.3 For $\mathbf{x} \in \ell_{1}$ the $\operatorname{set}\{n \in \mathbb{N}: x(n) \neq 0\}$ is called the support of $\mathbf{x}$. It is well known that if we take a sequence $\left(\mathbf{x}^{(i)}\right)_{i \in \mathbb{N}}$ in $\ell_{1}$ with pairwise disjoint supports and $\left\|\mathbf{x}^{(i)}\right\|=1$, then the closed subspace $V$ generated by $\left(\mathbf{x}^{(i)}\right)_{i \in \mathbb{N}}$ is of the form $V=\left\{t_{i} \mathbf{x}^{(i)}: t_{i} \in \ell_{1}\right\}$. To prove the spaceability of $\mathcal{A O S} \mathcal{S}_{u b}$ let us divide $\mathbb{N}$ into infinite number of infinite sets $N_{i}$. So $\mathbb{N}=\bigcup_{i=1}^{\infty} N_{i}$ and $N_{i} \cap N_{j}=\emptyset$. Let $\mathbf{b}=(b(n))$ be an arbitrary fixed sequence belonging to $\mathcal{A O S} \mathcal{S}_{u b}$ with $\|\mathbf{b}\|=1$. Let us define a family $\left(\mathbf{b}^{(i)}\right)_{i \in \mathbb{N}}$ by

$$
b^{(i)}(n)= \begin{cases}b(m) & \text { if } n \text { is the } m \text {-th element of } N_{i}, \\ 0 & \text { otherwise. }\end{cases}
$$

Let us observe, that the subspace $V_{b}$ generated by $\left(\mathbf{b}^{(i)}\right)_{i \in N}$ is contained in $\mathcal{A O S} \mathcal{S}_{u b}$. Indeed, we have $\lim \sup n b(n)=\infty$. Hence, for $N_{i}=\left\{n_{1}^{(i)}, n_{2}^{(i)}, \ldots, n_{m}^{(i)}, \ldots\right\}$ we have $t_{i} n_{m}^{(i)} b^{(i)}\left(n_{m}\right)=$ $t_{i} n_{m}^{(i)} b(m) \geq t_{i} m b(m)$. 
A proof of spaceability of $\mathcal{A O S}_{b}$ should be unfortunately more complicated. Let $N_{i}=\left\{2^{i-1}(2 n-1): n=1,2, \ldots\right\}$. (Then $N_{1}=\{1,3,5, \ldots\}, N_{2}=\{2,6,10, \ldots\}$, $N_{3}=\{3,12,20, \ldots\}$ and so on).

Let $A_{1}, A_{2}, \ldots$ be a sequence of subsets of $\mathbb{N}$ such that $\sum_{n \in A_{i}} \frac{1}{2 n-1}=2^{i-1}$. Let us define the sequences $\left(\mathbf{a}^{(i)}\right)_{i \in \mathbb{N}}$ by

$$
a^{(i)}(k)= \begin{cases}\frac{1}{2^{i-1}(2 n-1)} & \text { for } k=2^{i-1}(2 n-1) \text { and } n \in A_{i} \\ 0 & \text { otherwise. }\end{cases}
$$

Let us observe that $\left\|\mathbf{a}^{(i)}\right\|=1$ their supports are pariwise disjoint (the supports of $\mathbf{a}^{(i)}$ is contained in $N_{i}$ ) and the subspace $V_{a}$ generated by $\left(\mathbf{a}^{(i)}\right)_{i \in \mathbb{N}}$ is contained in $\mathcal{A O S} \mathcal{S}_{b}$. Indeed, for $\mathbf{x}=\sum_{i} t_{i} \mathbf{a}^{(i)}$ we have lim sup $|k x(k)|=\sup \left|t_{i}\right| \cdot\left|k a^{(i)}(k)\right|=\sup \left|t_{i}\right|$.

For similar constructions as the above one, see f.e. [2,6].

The next theorem shows, that set $\mathcal{A O S} \cap \mathcal{F} \mathcal{Z}$ is not spaceable.

Theorem 3.4 Let $Y \subset \ell_{1}$ be a closed, infinitely dimensional subspace. Then there exists $\mathbf{z} \in Y \backslash\{\mathbf{0}\}$ and infinite set $N \subset \mathbb{N}$ such that $z(n)=0$ for $n \in N$. In other words, $\mathcal{F} \mathcal{Z}$ is not spaceable.

Proof Let $\mathbf{y}_{0} \in Y \backslash\{\mathbf{0}\}$. There exists $n_{0} \in \mathbb{N}$ such that $y_{0}\left(n_{0}\right) \neq 0$. Without lost of generality we can assume that $y_{0}\left(n_{0}\right)>0$ and $\left\|\mathbf{y}_{0}\right\|=1$. Let us take $m_{0} \geq n_{0}$ such that

$$
\sum_{n=m_{0}+1}^{\infty}\left|y_{0}(n)\right|<\frac{1}{4} \text {. }
$$

The subspace $X_{0}=\left\{\mathbf{x} \in \ell_{1}: x(n)=0\right.$ for $\left.n \leq m_{0}\right\}$ has the finite co-dimension so the subspace $Y_{1}=Y \cap X_{0}$ is infinitely dimensional. Let $\mathbf{y}_{1} \in Y_{1}$ and

$$
\left\|\mathbf{y}_{1}\right\|=\sum_{n=m_{0}+1}^{\infty}\left|y_{1}(n)\right|=\frac{1}{2} \text {. }
$$

By (1) and (2) we can find $n_{1}>m_{0}$ such that $\left|y_{1}\left(n_{1}\right)\right|>0$ and $\left|y_{1}\left(n_{1}\right)\right| \geq\left|y_{0}\left(n_{1}\right)\right|$. We may assume that $y_{1}\left(n_{1}\right) y_{0}\left(n_{1}\right) \geq 0$. Let us put

$$
c_{1}:=\frac{y_{0}\left(n_{1}\right)}{y_{1}\left(n_{1}\right)} \in[0,1], \quad \mathbf{z}_{0}:=\mathbf{y}_{0} \quad \text { and } \quad \mathbf{z}_{1}:=\mathbf{y}_{0}-c_{1} \mathbf{y}_{1} .
$$

Then

(1) $z_{1}\left(n_{0}\right)=y_{0}\left(n_{0}\right) \neq 0$;

(2) $z_{1}\left(n_{1}\right)=y_{0}\left(n_{1}\right)-y_{0}\left(n_{1}\right)=0$;

(3) $\left\|\mathbf{z}_{1}-\mathbf{z}_{0}\right\|=c_{1}\left\|\mathbf{y}_{1}\right\| \leq 1 / 2$.

Let us choose $m_{1} \geq n_{1}$ such that

$$
\sum_{n=m_{1}+1}\left|z_{1}(n)\right|<\frac{1}{8}
$$

Let us put $X_{1}=\left\{\mathbf{x} \in \ell_{1}: x(n)=0\right.$ for $\left.n \leq m_{1}\right\}$ and $Y_{2}=Y_{1} \cap X_{1}$. Then $\operatorname{codim}\left(X_{1}\right)<\infty$ and hence $\operatorname{dim}\left(Y_{2}\right)=\infty$. Let $\mathbf{y}_{2} \in Y_{2}$ and $\left\|\mathbf{y}_{2}\right\|=1 / 4$. We can find $n_{2}>m_{1}>n_{1}$ such that $y_{2}\left(n_{2}\right) \neq 0$ and $\left|y_{2}\left(n_{2}\right)\right| \geq\left|z_{1}\left(n_{2}\right)\right|$. We may assume that $y_{2}\left(n_{2}\right) \cdot z_{1}\left(n_{2}\right) \geq 0$. Let us put

$$
c_{2}:=\frac{z_{1}\left(n_{2}\right)}{y_{2}\left(n_{2}\right)} \text { and } \quad \mathbf{z}_{2}=\mathbf{z}_{1}-c_{2} \mathbf{y}_{2} \text {. }
$$


Then we have

(1) $z_{2}\left(n_{0}\right)=z_{1}\left(n_{0}\right)-c_{2}\left(y_{2}\left(n_{0}\right)\right)=y_{0}\left(n_{0}\right) \neq 0$;

(2) $z_{2}\left(n_{1}\right)=z_{2}\left(n_{2}\right)=0$;

(3) $\left\|\mathbf{z}_{2}-\mathbf{z}_{1}\right\|=c_{2}\left\|\mathbf{y}_{2}\right\| \leq\left\|\mathbf{y}_{2}\right\|=1 / 4$.

In the next steps we define inductively a sequence $\left(\mathbf{z}_{k}\right)$, an increasing sequence $\left(n_{k}\right)$ and a sequence $\left(c_{k}\right)$ where $c_{k} \in[0,1)$ for $k=1,2, \ldots$, such that:

(1) $z_{k}\left(n_{0}\right)=z_{k-1}\left(n_{0}\right)-c_{k}\left(y_{k}\left(n_{0}\right)\right)=y_{0}\left(n_{0}\right) \neq 0$;

(2) $z_{k}\left(n_{k-1}\right)=z_{k}\left(n_{k}\right)=0$;

(3) $\left\|\mathbf{z}_{k}-\mathbf{z}_{k-1}\right\|=c_{k}\left\|\mathbf{y}_{k}\right\| \leq\left\|\mathbf{y}_{k}\right\|=1 / 2^{k}$.

By (3) the sequence $\left(\mathbf{z}_{k}\right)$ is Cauchy sequence in $Y \subset \ell_{1}$. Hence it is convergent in $\ell_{1}$ to some element $\mathbf{z}$ for which $z\left(n_{0}\right) \neq 0$ and $z\left(n_{i}\right)=0$ for all $i \geq 1$.

The proof of nonspaceability of some other sets in $\ell_{1}$ can be found in [3].

\section{Topological properties}

In the previous section we have shown that the set of all sequences from $\ell_{1}$, for which the assertion of Olivier's theorem does not hold is algebraically large. We will show now that it is also large in the topological sense. Let us recall that $\mathcal{A O S}:=\left\{\mathbf{a} \in \ell_{1}: n a(n) \not \rightarrow 0\right\}$ and $\mathcal{F} \mathcal{Z}$ denotes the set of all sequences from $\ell_{1}$ which have at most finitely many zeros.

Theorem 4.1 $\mathcal{A O S} \cap \mathcal{F} \mathcal{Z}$ is $G_{\delta \sigma}$ but not $F_{\sigma \delta}$ subset of $\ell_{1}$. Moreover $\mathcal{A O S} \cap \mathcal{F Z}$ contains a dense $G_{\delta}$, and therefore is comeager in $\ell_{1}$.

Proof Firstly, we observe that $\mathcal{A O S} \cap \mathcal{F} \mathcal{Z}$ is $G_{\delta \sigma}$. We have

$$
\begin{aligned}
& \mathcal{A O S} \cap \mathcal{F Z}=\left\{\mathbf{a} \in \ell_{1}: \exists m \forall n \exists k>n(|k a(k)|>1 / m) \text { and } \exists n \forall k>n(a(k) \neq 0)\right\} \\
& =\bigcup_{m \in \mathbb{N}} \bigcap_{n \in \mathbb{N}} \bigcup_{k>n}\left\{\mathbf{a} \in \ell_{1}:|k a(k)|>1 / m\right\} \cap \bigcup_{n \in \mathbb{N}} \bigcap_{k>n}\left\{\mathbf{a} \in \ell_{1}: a(k) \neq 0\right\} .
\end{aligned}
$$

Since $\left\{\mathbf{a} \in \ell_{1}:|k a(k)|>1 / m\right\}$ and $\left\{\mathbf{a} \in \ell_{1}: a(k) \neq 0\right\}$ are open, the set $\mathcal{A O S} \cap \mathcal{F} \mathcal{Z}$ is $G_{\delta \sigma}$.

Now, we observe that $\mathcal{A O S} \cap \mathcal{F Z}$ contains a dense $G_{\delta}$. Put

$$
G:=\left\{\mathbf{a} \in \ell_{1}: \forall n \exists k>n(|k a(k)|>1) \text { and } \forall k(a(k) \neq 0)\right\} .
$$

Clearly $G$ is a subset of $\mathcal{A O S} \cap \mathcal{F Z}$. Using the similar reasoning as in the previous paragraph, we obtain that $G$ is $G_{\delta}$. It remains to prove that $G$ is dense in $\ell_{1}$. To this end fix $\varepsilon>0$ and $\mathbf{b} \in \ell_{1}$. We will construct $\mathbf{a} \in G$ with $\|\mathbf{a}-\mathbf{b}\| \leq \varepsilon$. Let us define $\mathbf{c} \in \ell_{1}$ as follows

$$
c(n)= \begin{cases}b(n), & \text { if } b(n) \neq 0 \\ \frac{\varepsilon}{2^{n+1}}, & \text { if } b(n)=0 .\end{cases}
$$

Then $\|\mathbf{c}-\mathbf{b}\| \leq \varepsilon / 2$ and the sequence $\mathbf{c}$ has no zeros. Let us fix a strictly increasing sequence of indices $\left(k_{i}\right)$ with $\frac{\varepsilon}{2^{i+1}}>\frac{1}{k_{i}}$, and put $K:=\left\{k_{i}: i \in \mathbb{N}\right\}$. We define a as follows

$$
a(k)= \begin{cases}c(k), & \text { if } k \notin K \\ c(k), & \text { if } k=k_{i} \in K \text { and }\left|c\left(k_{i}\right)\right|>\frac{\varepsilon}{2^{i+1}} \\ c(k)+\frac{\varepsilon}{2^{i+1}} \cdot \operatorname{sgn}(c(k)), & \text { if } k=k_{i} \in K \text { and }\left|c\left(k_{i}\right)\right| \leq \frac{\varepsilon}{2^{i+1}} .\end{cases}
$$


By the construction a has no zeros. For any $n \in \mathbb{N}$ one can find $k_{i}>n$, and $k_{i}\left|a\left(k_{i}\right)\right|>$ $k_{i} \cdot \frac{\varepsilon}{2^{i+1}}>k_{i} \cdot \frac{1}{k_{i}}=1$. Therefore $\mathbf{a} \in G$. Note that

$$
\|\mathbf{a}-\mathbf{b}\| \leq\|\mathbf{a}-\mathbf{c}\|+\|\mathbf{c}-\mathbf{b}\| \leq \sum_{n=1}^{\infty}|a(n)-c(n)|+\frac{\varepsilon}{2} \leq \frac{\varepsilon}{2}+\frac{\varepsilon}{2}=\varepsilon .
$$

Finally, we show that $\mathcal{A O S} \cap \mathcal{F} \mathcal{Z}$ is not $F_{\sigma \delta}$. Put

$$
D=\left\{\mathbf{x} \in \mathbb{N}^{\mathbb{N}}: \liminf _{n \rightarrow \infty} x(n)<\infty\right\} .
$$

This is a $G_{\delta \sigma}$ subset of $\mathbb{N}^{\mathbb{N}}$ considered with the product topology which is not $F_{\sigma \delta}$, see [11, Section 23A]. Our aim is to construct a continuous function $f: \mathbb{N}^{\mathbb{N}} \rightarrow \ell_{1}$ such that $f^{-1}[\mathcal{A O S} \cap \mathcal{F} \mathcal{Z}]=D$. That would show that $\mathcal{A O S} \cap \mathcal{F} \mathcal{Z}$ is not $F_{\sigma \delta}$ as $D$ is not $F_{\sigma \delta}$ and continuous preimages preserve Borel pointclasses.

Let us fix a strictly increasing sequence $\left(k_{n}\right) \in \mathbb{N}^{\mathbb{N}}$ with $\sum_{i=1}^{\infty} \frac{1}{k_{i}}<\infty$. Let us define $f: \mathbb{N}^{\mathbb{N}} \rightarrow \ell_{1}$ as follows

$$
f(\mathbf{x})(n)= \begin{cases}\frac{1}{2^{n}}, & \text { if } n \notin\left\{k_{i}: i \in \mathbb{N}\right\} \\ \frac{1}{k_{i} x(i)}, & \text { if } n=k_{i} \text { for some } i\end{cases}
$$

First, let us note that

$$
\|f(\mathbf{x})\|=\sum_{n \notin\left\{k_{i}: i \in \mathbb{N}\right\}} \frac{1}{2^{n}}+\sum_{i=1}^{\infty} \frac{1}{k_{i} x(i)} \leq 2+\sum_{i=1}^{\infty} \frac{1}{k_{i}}<\infty,
$$

that is $f(\mathbf{x}) \in \ell_{1}$, which means that $f$ is well-defined. To prove that $f$ is continuous let us assume that $\mathbf{x}_{n} \rightarrow \mathbf{x}$ in $\mathbb{N}^{\mathbb{N}}$. Then

$$
\forall m \exists k \forall n \geq k \forall i \leq m \quad\left(x_{n}(i)=x(i)\right),
$$

which means that for any $m$ sequences $\mathbf{x}_{n}$ eventually equal to $\mathbf{x}$ up to $m$-th coordinate. Let $\varepsilon>0$. Find $i_{0}$ such that $\sum_{i \geq i_{0}} \frac{1}{k_{i}}<\varepsilon$. Let $m:=k_{i_{0}}-1$. find $k$ with

$$
\forall n \geq k \forall i \leq m\left(x_{n}(i)=x(i)\right) .
$$

Then

$$
\left\|f\left(\mathbf{x}_{n}\right)-f(\mathbf{x})\right\|=\sum_{i \geq i_{0}}\left|\frac{1}{k_{i} x_{n}(i)}-\frac{1}{k_{i} x(i)}\right|=\sum_{i \geq i_{0}} \frac{1}{k_{i}} \cdot\left|\frac{1}{x_{n}(i)}-\frac{1}{x(i)}\right| \leq \sum_{i \geq i_{0}} \frac{1}{k_{i}}<\varepsilon .
$$

Let us observe that $n f(\mathbf{x})(n) \rightarrow 0 \Longleftrightarrow k_{n} f(\mathbf{x})\left(k_{n}\right) \rightarrow 0$. It follows from the fact that $n / 2^{n} \rightarrow 0$.

Assume that $\mathbf{x} \in D$. Then $\lim \inf _{n \rightarrow \infty} x(n)=M<\infty$. Then there is a strictly increasing sequence of indices $\left(n_{l}\right)$ with $x\left(n_{l}\right)=M$ for every $l$. Then $f(\mathbf{x})\left(k_{n_{l}}\right)=\frac{1}{x\left(n_{l}\right) k_{n_{l}}}$, and consequently $\lim _{l \rightarrow \infty} k_{n_{l}} f(\mathbf{x})\left(k_{n_{l}}\right)=\frac{1}{M} \neq 0$. Therefore $n f(\mathbf{x})(n) \not \rightarrow 0$, which means that $f(\mathbf{x}) \in \mathcal{A O S} \cap \mathcal{F Z}$.

Now, assume that $\mathbf{x} \notin D$. Then $\lim _{n \rightarrow \infty} x(n)=\infty$. Note that

$$
\lim _{n \rightarrow \infty} k_{n} f(\mathbf{x})\left(k_{n}\right)=\lim _{n \rightarrow \infty} k_{n} \cdot \frac{1}{k_{n} x(n)}=\lim _{n \rightarrow \infty} \frac{1}{x(n)}=0 .
$$

By our observation this implies $n f(\mathbf{x})(n) \rightarrow 0$, which means $f(\mathbf{x}) \notin \mathcal{A O S} \cap \mathcal{F} \mathcal{Z}$.

The last two paragraphs show that $f^{-1}[\mathcal{A O S} \cap \mathcal{F} \mathcal{Z}]=D$. This finishes the proof. 
Open Access This article is licensed under a Creative Commons Attribution 4.0 International License, which permits use, sharing, adaptation, distribution and reproduction in any medium or format, as long as you give appropriate credit to the original author(s) and the source, provide a link to the Creative Commons licence, and indicate if changes were made. The images or other third party material in this article are included in the article's Creative Commons licence, unless indicated otherwise in a credit line to the material. If material is not included in the article's Creative Commons licence and your intended use is not permitted by statutory regulation or exceeds the permitted use, you will need to obtain permission directly from the copyright holder. To view a copy of this licence, visit http://creativecommons.org/licenses/by/4.0/.

\section{References}

1. Aron, R.M., Bernal González, L., Pellegrino, D.M., Seoane Sepúlveda, J.B.: Lineability: The Search for Linearity in Mathematics. Monographs and Research Notes in Mathematics, CRC Press, Boca Raton (2016)

2. Banakh, T., Bartoszewicz, A., Głąb, S., Szymonik, E.: Algebraic and topological properties of some sets in $\ell_{1}$. Colloq. Math. 129(1), 75-85 (2012)

3. Banakh, T., Bartoszewicz, A., Głąb, S., Szymonik, E.: Erratum to "Algebraic and topological properties of some sets in $\ell_{1}$ " (Colloq. Math. 129 (2012), 75-85). Colloq. Math. 135(2), 295-298 (2014)

4. Bartoszewicz, A., Głąb, S.: Strong algebrability of sets of sequences and functions. Proc. Am. Math. Soc. 141, 827-835 (2013)

5. Bernal-González, L., Pellegrino, D., Seoane-Sepúlveda, J.B.: Linear subsets of nonlinear sets in topological vector spaces. Bull. Am. Math. Soc. (N.S.) 51(1), 71-130 (2014)

6. Bernal-González, L., Cabrera, O.: Manuel spaceability of strict order integrability. J. Math. Anal. Appl. 385(1), 303-309 (2012)

7. Faisant, Alain, Grekos, Georges, Mišík, L.: Some generalizations of Olivier's theorem. Math. Bohem. 141(4), 483-494 (2016)

8. Gogola, J., Mačaj, M., Visnyai, T.: On $\mathcal{I}_{c}^{(q)}$-convergence. Ann. Math. Inform. 38, 27-36 (2011)

9. Kostyrko, P., Šalát, T., Wilczyński, W.: I-convergence. Real Anal. Exch. 26(2), 669-685 (2000/2001)

10. Krzyż, J.: Olivier's theorem and its generalizations. (Polish) Prace Mat. 2, 159-164 (1956)

11. Kechris, A.S.: Classical Descriptive Set Theory. Graduate Texts in Mathematics, vol. 156. Springer, New York (1995)

12. Mišík, L., Tóth, J.: Ideal extension of Olivier's theorem. Real Anal. Exch. 46(1)(2021). https://doi.org/ 10.14321/realanalexch.46.1.0001

13. Olivier, M.: Louis Remarques sur les séries infinies et leur convergence, (French). J. Reine Angew. Math. 2, 31-44 (1827)

14. Šalát, T., Toma, V.: A classical Olivier's theorem and statistical convergence. Ann. Math. Blaise Pascal 10(2), 305-313 (2003)

15. de la Vallée Poussin, Ch.J.: Cours d'Analyse Infinitésimale, (French), vol. I. Dover Publications, New York (1946)

Publisher's Note Springer Nature remains neutral with regard to jurisdictional claims in published maps and institutional affiliations. 